\title{
COVERING LEMMAS \\ AND AN APPLICATION TO NODAL GEOMETRY \\ ON RIEMANNIAN MANIFOLDS
}

\author{
GUOZHEN LU
}

(Communicated by J. Marshall Ash)

\begin{abstract}
The main part of this note is to show a general covering lemma in $R^{n}, n \geq 2$, with the aim to obtain the estimate for BMO norm and the volume of a nodal set of eigenfunctions on Riemannian manifolds.
\end{abstract}

\section{INTRODUCTION}

This article is a continuation of our previous work [L]. In [L] we proved a covering lemma in $R^{2}$ and applied it to the BMO norm estimates for eigenfunctions on Riemannian surfaces. The principal part of this article is to prove a general covering lemma in $R^{n}$ for $n \geq 2$. As applications, we can obtain the BMO estimate for eigenfunctions and the volume estimate for the nodal set.

Let $M^{n}$ be a smooth, compact, and connected Riemannian manifold with no boundary. Let $\Delta$ denote the Laplacian on $M^{n}$. Let $-\Delta u=\lambda u, u$ an eigenfunction with eigenvalue $\lambda, \lambda>1$.

Our main results can be stated as follows

Theorem A (BMO estimate for $\log |u|)$. For $u, \lambda$ as above and $n \geq 3$,

$$
\|\log |u|\|_{\mathrm{BMO}} \leq C \lambda^{n-1 / 8}(\log \lambda)^{2},
$$

where $C$ is independent of $\lambda$ and $u$ and is only dependent on $n$ and $M^{n}$.

Theorem B (geometry of nodal domains). Let $n \geq 3$ and $u, \lambda$ as above. Let $B \subset M^{n}$ be any ball, and let $\Omega \subset B$ be any of the connected components of $\{x \in B: u(x) \neq 0\}$. If $\Omega$ intersects the middle half of $B$, then

$$
|\Omega| \geq C \lambda^{-2 n^{2}-n / 4}(\log \lambda)^{-4 n}|B|,
$$

where $C$ is independent of $\lambda$ and $u$.

Donnelly and Fefferman [DF1, DF2] and Chanillo and Muckenhoupt [CM] proved Theorem A with $\lambda^{n-1 / 8}(\log \lambda)^{2}$ replaced by $\lambda^{n(n+2) / 4}$ and $\lambda^{n} \log \lambda$, respectively, and Theorem B with $\lambda^{-2 n^{2}-n / 4}(\log \lambda)^{-4 n}$ replaced by $\lambda^{-\left(n+n^{2}(n+2)\right) / 2}$ and $\lambda^{-2 n^{2}-n / 2}(\log \lambda)^{-2 n}$, respectively.

Received by the editors June 28, 1991.

1991 Mathematics Subject Classification. Primary 35B05, $58 \mathrm{G} 03$. 
In the case $n=2$, the following has been proved in [L]:

$$
\|\log u\|_{\mathrm{BMO}} \leq C \lambda^{15 / 8+\varepsilon},
$$

where $\varepsilon>0$ and $C=C(\varepsilon)$ is independent of $\lambda$ and $u$.

The proof of Theorems A and B is based on the following covering lemma, which is of its own right and is really the main result of this paper.

Lemma $\mathrm{C}$ (covering lemma in $R^{n}$ ). Let $\delta>0$ be small enough. Let $\left\{B_{\alpha}\right\}_{\alpha \in I}$ be any finite collection of balls in $R^{n} \quad(n \geq 2)$. Then one can select a subcollection $B_{1}, B_{2}, \ldots, B_{N}$ such that

(1) $\bigcup_{\alpha} B_{\alpha} \subset \bigcup_{i=1}^{N}(1+\delta) B_{i}$,

(2) $\sum_{i=1}^{N} \chi_{B_{i}}(x) \leq C\left(\log \frac{1}{\delta}\right) \delta^{-n+1 / 4}$ for all $x \in R^{n}$,

where $C$ depends only on $n$ but is independent of $\delta$ and the given balls.

From the proof of Lemma $C$ (see $\S 3$ ), we can see that we also have covering lemmas for any finite collection of balls with some restrictions on the lower and upper bounds for the radii of the given balls. We state here these results for the interested reader.

Lemma $\mathbf{D}$ (for balls with almost equal radii). Let $\delta$ be small enough. Let $\left\{B_{\alpha}\right\}_{\alpha \in I}$ be any finite collection of balls in $R^{n}(n \geq 2)$ with $r \leq \rho\left(B_{\alpha}\right) \leq$ $r+2^{k} \delta$ for some $2^{k} \leq r \leq 2^{k+1}$, where $k$ is an integer. Then one can select a subcollection of balls $B_{1}, \ldots, B_{N}$ such that

$$
\begin{gathered}
\bigcup_{\alpha} B_{\alpha} \subset \bigcup_{i=1}^{N}(1+\delta) B_{i}, \\
\sum_{i=1}^{N} \chi_{B_{i}}(x) \leq C \delta^{-(n-3 / 4)} \text { for all } x \in R^{n},
\end{gathered}
$$

where $C$ only depends on the dimension $n$ but is independent of $k, \delta$, and the given balls.

The proof of Lemma D will be based on Lemma 3.1 in $\S 3$. The method of proof is similar to the one of Lemma 3.7.

Lemma $\mathbf{E}$ (for balls with radii of lower and upper bounds). Let $\delta$ be small enough and $k$ be any integer. Let $\left\{B_{\alpha}\right\}_{\alpha \in I}$ be any finite collection of balls in $R^{n}, n \geq 2$, with $2^{k} \leq \rho\left(B_{\alpha}\right) \leq 2^{k+1}$. Then one can select a subcollection $B_{1}, \ldots, B_{N}$ such that

$$
\begin{gathered}
\bigcup_{\alpha} B_{\alpha} \subset \bigcup_{i=1}^{N}(1+\delta) B_{i}, \\
\sum_{i=1}^{N} \chi_{B_{i}}(x) \leq C \delta^{-(n-1 / 4)} \text { for all } x \in R^{n},
\end{gathered}
$$

where $C$ depends only on the dimension $n$ but is independent of $k, \delta$, and the given balls.

Lemma $\mathrm{E}$ is just a restatement of Lemma 3.7 in $\S 3$ by replacing $\left(1+C_{n}\right) \delta$ by $(1+\delta)$. 
This covering Lemma $\mathrm{C}$ is an improvement of the one in [CM], which was $\delta^{-n}$ on the right-hand side of (2). This type of covering lemma may be useful since the Vitali covering lemma is not good enough in many cases and the Besicovitch covering lemma does not apply sometimes (see [SW]). The proof of the corresponding covering lemma in $[\mathrm{CM}]$ is very elegant, but the covering lemma in [CM] does not have the implications of Lemmas D and E. Obviously, from the proof of Lemma $C$, we can see that this covering lemma is not the best possible.

Once we have Lemma $C$, we can just modify the proof given in [CM], and thus the proof of Theorems A and B will be omitted here. The interested reader should refer to [CM]. Instead, we will concentrate on the proof of the covering lemmas, which will be given in the next two sections.

Notation. Throughout this paper, we will denote by $c$ or $C$ the generic constants not exactly equal at each occurrence and which depend on the dimension only. We will also use $\rho(B)$ to denote the radius of the ball $B$. If $B$ is an $(n+1)$-dimensional ball in $R^{n+1}$, then we denote by $B^{*}$ the projection of $B$ onto the $n$-dimensional hyperplane $\left\{x_{n+1}=0\right\}$. Obviously, $B^{*}$ is an $n$-dimensional ball with $\rho(B)=\rho\left(B^{*}\right)$.

\section{A BASIC COVERING LEMMA IN $R^{n}$}

The main goal of this section is to prove a basic covering lemma in $R^{n}$ for balls whose radii are close to one another and centered in an $n$-dimensional cube with sidelength $\sqrt{\delta}$. As mentioned in $\S 1$, we will denote by $B^{*}$ the projected ball of the $(n+1)$-dim ball $B \subset R^{n+1}$ to the hyperplane $\left\{x_{n+1}=0\right\}$.

Since the proof of Lemma $C$ adapts the method of induction on the dimension $n$ on each cube with sidelength $\sqrt{\delta}$ and is based on a basic covering lemma in $R^{2}$ proved in [L, Lemma 4.1], we recall this essential lemma first.

Lemma 2.1. Let $\delta>0$ be given small enough. Given any cube $\mathscr{Q}$ in $R^{2}$ with sidelength $\sqrt{\delta}$ and given any finite collection of balls $\left\{B_{\alpha}\right\}_{\alpha \in I}$ in $R^{2}$ with $r \leq$ $\rho\left(B_{\alpha}\right) \leq r+\delta$, for some $1 \leq r \leq 2$, and centered in this cube $\mathscr{Q}$, one can select a subcollection of balls $B_{1}, \ldots, B_{N}$ such that

$$
\begin{gathered}
\bigcup_{\alpha} B_{\alpha} \subset \bigcup_{i=1}^{N}(1+\delta) B_{i}, \\
N \leq c \delta^{-1 / 4},
\end{gathered}
$$

where $c$ is an absolute constant independent of $\delta$ and the given balls.

We also need the following.

Lemma 2.4. Let $\delta>0$ be given small enough. Let $B_{1}$ and $B_{2}$ be two $(m+1)$ dimensional balls in $R^{m+1}$ with radius $r \leq \rho\left(B_{i}\right) \leq r+\delta$ for some $1 \leq r \leq 2$ $(i=1,2)$. Assume that $B_{1}$ is centered at the origin in $R^{m+1}$ and $B_{2}$ is centered at $O_{2}=\left(s_{1}, s_{2}, \ldots, s_{m}, s_{m+1}\right)$ such that $\sqrt{\sum_{i=1}^{m+1} s_{i}^{2}} \leq \sqrt{(m+1) \delta}$ and $\left|s_{m+1}\right| \leq \delta$. If the point, lying on the boundary of $B_{1}, A=\left(0, \ldots, 0, \rho\left(B_{1}\right), 0\right)$ $\in\left(1+C_{m} \delta\right) B_{2}^{*}$, then all those points inside the ball $B_{1}$ of the form $\left(0, \ldots, 0, t_{m}, t_{m+1}\right)$ with $t_{m} \geq 0$ (we call the set of these points $P$ ) are in 
the ball $\left(1+C_{m+1} \delta\right) B_{2}$, where $C_{m}$ and $C_{m+1}$ are two constants only depending on $m$.

Proof. Since $A \in\left(1+C_{m} \delta\right) B_{2}^{*}$, the distance between $A$ and the center of $B_{2}^{*}$, i.e., $\left(s_{1}, s_{2}, \ldots, s_{m}, 0\right)$, is no more than $\left(1+C_{m} \delta\right) \rho\left(B_{2}\right)$. This implies that

$$
s_{1}^{2}+\cdots+s_{m-1}^{2}+\left(s_{m}-\rho\left(B_{1}\right)\right)^{2} \leq\left[\left(1+C_{m} \delta\right) \rho\left(B_{2}\right)\right]^{2} .
$$

By the hypothesis, we have $s_{1}^{2}+\cdots+s_{m+1}^{2} \leq(m+1) \delta,\left|s_{m+1}\right| \leq \delta$. We also have that $t_{m}^{2}+t_{m+1}^{2} \leq \rho\left(B_{1}\right)^{2}$ and $t_{m} \geq 0$ for the points $\left(0, \ldots, 0, t_{m}, t_{m+1}\right)$ in $P$. We now claim that $s_{m} \geq-C \delta$ for some $C=C(m)$. In fact, from (2.5), noting $\left|s_{m}-\rho\left(B_{1}\right)\right| \leq\left(1+C_{m} \delta\right) \rho\left(B_{2}\right)$, it follows that $s_{m} \geq-\left(1+C_{m}\right) \delta \rho\left(B_{2}\right)+\rho\left(B_{1}\right) \geq$ $-C \delta$ by the hypothesis that $r \leq \rho\left(B_{i}\right) \leq r+\delta$ for some $1 \leq r \leq 2(i=1,2)$.

Thus the distance between $O_{2}$ and the points in $P$ are not more than

$$
\begin{aligned}
s_{1}^{2}+ & s_{2}^{2}+\cdots+s_{m-1}^{2}+\left(s_{m}-t_{m}\right)^{2}+\left(s_{m+1}-t_{m+1}\right)^{2} \\
& =\sum_{i=1}^{m-1} s_{i}^{2}+s_{m}^{2}+s_{m+1}^{2}+t_{m}^{2}+t_{m+1}^{2}-2 s_{m} t_{m}-2 s_{m+1} t_{m+1} \\
& \leq(m+1) \delta+\rho\left(B_{1}\right)^{2}+C \delta \leq\left[\left(1+C_{m+1} \delta\right) \rho\left(B_{2}\right)\right]^{2} .
\end{aligned}
$$

Thus $P \subset\left(1+C_{m+1} \delta\right) B_{2}$. Q.E.D.

Remark. The above set $P$ is actually the intersection points between the ball $B_{1}$ and the hyperplane $\left\{x_{1}=0, \ldots, x_{m-1}=0\right\}$.

Lemma 2.6. Let $\delta>0$ be given small enough. Let $\mathscr{Q}=\left\{\left(x_{1}, \ldots, x_{n}, x_{n+1}\right)\right.$ : $0 \leq x_{i} \leq \sqrt{\delta}$, for $\left.1 \leq i \leq n, 0 \leq x_{n+1} \leq \delta\right\}$ be the parallelopiped in $R^{n+1}$. Assume as given any finite collection of $(n+1)$-dimensional balls $\left\{B_{\alpha}\right\}_{\alpha \in I}$ in $R^{n+1}$ with $r \leq \rho\left(B_{\alpha}\right) \leq r+\delta$, for some $1 \leq r \leq 2$, centered in this parallelopiped Q. Assume that there exists a subcollection of balls $B_{1}, \ldots, B_{N}$ such that the projected balls $\left\{B_{i}^{*}\right\}_{i=1}^{N}$ onto the hyperplane $\left\{x_{n+1}=0\right\}$ satisfy

$$
\bigcup_{\alpha} B_{\alpha}^{*} \subset \bigcup_{i=1}^{N}\left(1+C_{n} \delta\right) B_{i}^{*} .
$$

Then we have

$$
\bigcup_{\alpha} B_{\alpha} \subset \bigcup_{i=1}^{N}\left(1+C_{n+1} \delta\right) B_{i}
$$

where $C_{n+1}$ only depends on the dimension $n$ and is independent of $\delta$ and the given balls.

Proof. Fix $B_{\alpha}$ and let $x \in B_{\alpha}$. Let $O_{\alpha}=\left(t_{1}, \ldots, t_{n+1}\right)$ be the center of $B_{\alpha}$ and $O_{\alpha}^{*}$ and $x^{*}$ be the projections of $O_{\alpha}$ and $x$ onto the hyperplane $x_{n+1}=0$ respectively. Let $l_{\alpha}$ be the ray originating from $O_{\alpha}$ and passing through $x^{*}$, and let $A_{\alpha}$ be the intersection point between $l_{\alpha}$ and the boundary $\partial B_{\alpha}^{*}$ of $B_{\alpha}^{*}$. On account of (2.7), we have $A_{\alpha} \in\left(1+C_{n} \delta\right) B_{i}^{*}$ for some $i$, and we assume the coordinate of the center of $B_{i}$ is $O_{i}=\left(s_{1}, \ldots, s_{n+1}\right)$. Then we claim $x \in\left(1+C_{n+1} \delta\right) B_{i}$ for the same $i$. To show the claim, we adapt the new Cartesian coordinates $\left(x_{1}^{\prime}, \ldots, x_{n}^{\prime}, x_{n+1}^{\prime}\right)$ such that

(1) $\left(x_{1}^{\prime}, \ldots, x_{n}^{\prime}, x_{n+1}^{\prime}\right)$ is derived from $\left(x_{1}, \ldots, x_{n}, x_{n+1}\right)$ by an orthogonal transformation and a translation. 
(2) The origin of the new coordinate is at $O_{\alpha}$ and the coordinate of $A_{\alpha}$ is of the form $\left(0, \ldots, 0, \rho\left(B_{\alpha}\right), 0\right)$. Then by the rigid invariance of the distances, all the $(n+1)$-dimensional balls $B_{\alpha}$ are still balls with the same radii under the new coordinates $\left(x_{1}^{\prime}, \ldots, x_{n}^{\prime}, x_{n+1}^{\prime}\right)$.

(3) Since the distance between $O_{\alpha}$ and $O_{i}$ is no more than $\sqrt{(n+1) \delta}$ and $\left|s_{n+1}-t_{n+1}\right| \leq \delta$, the new coordinate of $O_{i}, O_{i}=\left(s_{1}^{\prime}, \ldots, s_{n+1}^{\prime}\right)$, satisfies $\sqrt{\sum_{i=1}^{n+1}\left(s_{i}^{\prime}\right)^{2}} \leq \sqrt{(n+1) \delta}$ and $\left|s_{n+1}^{\prime}\right| \leq \delta$.

Thus, by Lemma 2.4, there exists a constant $C_{n+1}$ independent of $\delta$ and the given balls, only dependent on the dimension $n$, such that $x \in$ $\left(1+C_{n+1} \delta\right) B_{i}$. Since $x \in B_{\alpha}$ is arbitrary and $B_{\alpha}$ is an arbitrary ball in $\left\{B_{\alpha}\right\}$, we are done. Q.E.D.

By using Lemma 2.6, we can show the following basic covering lemma in $R^{n}, n \geq 2$.

Lemma 2.8. Let $\delta>0$ be given small enough. Given any cube $\mathscr{Q}_{n}$ in $R^{n}$ with sidelength $\sqrt{\delta}$ and any finite collection of balls $\left\{B_{\alpha}\right\}_{\alpha \in I}$ in $R^{n}$ with $r \leq$ $\rho\left(B_{\alpha}\right) \leq r+\delta$, for some $1 \leq r \leq 2$, and centered in this cube $\mathscr{Q}_{n}$, one can select a subcollection of balls $B_{1}, \ldots, B_{N}$ such that

$$
\begin{gathered}
\bigcup_{\alpha} B_{\alpha} \subset \bigcup_{i=1}^{N}\left(1+C_{n} \delta\right) B_{i}, \\
N \leq c \delta^{-(2 n-3) / 4},
\end{gathered}
$$

where $C_{n}$ only depends on the dimension $n$ and is independent of $\delta$ and the given balls and $c$ is a fixed constant which is equal to the constant $c$ in (2.3) of Lemma 2.1.

Proof. We have proved this lemma for the case $n=2$. Assume that (2.9) and (2.10) are true when $n=m$. We wish to prove the result for the case $n=m+1$.

Given a cube $Q_{m+1}=\left\{0 \leq x_{i} \leq \sqrt{\delta}: 1 \leq i \leq m+1\right\} \subset R^{m+1}$, we subdivide $Q_{m+1}$ into equal parallelopipeds

$$
Q_{m+1}^{k}=\left\{0 \leq x_{i} \leq \sqrt{\delta} \text { for } 1 \leq i \leq m, k \delta \leq x_{m+1} \leq(k+1) \delta\right\}
$$

for $k=0,1, \ldots, l$. Then $l \approx \delta^{-1 / 2}$.

Let $J_{k}=\left\{\alpha \in I: O_{\alpha} \in Q_{m+1}^{k}\right\}$, where $O_{\alpha}$ is the center of $B_{\alpha}$. We claim that given any finite collection of $(m+1)$-dimensional balls $\left\{B_{\alpha}\right\}$ with centers $O_{\alpha}$ in $Q_{m+1}^{k}$, we can select a subcollection of balls $\left\{B_{k_{j}}\right\}_{j=1}^{N_{k}}$ such that

$$
\begin{gathered}
\bigcup_{\alpha \in J_{k}} B_{\alpha} \subset \bigcup_{j=1}^{N_{k}}\left(1+C_{m+1} \delta\right) B_{k_{j}}, \\
N_{k} \leq c \delta^{-(2 m-3) / 4} .
\end{gathered}
$$

Without loss of generality, we only need to prove our claim for $Q_{m+1}^{0}$. We project the balls $B_{\alpha}$ to the $m$-dimensional hyperplane $x_{m+1}=0$. Then we obtain the corresponding $m$-dimensional balls $B_{\alpha}^{*}$ with centers $O_{\alpha}^{*}$ contained 
in the cube $Q_{m}=\left\{0 \leq x_{i} \leq \sqrt{\delta}, x_{m+1}=0\right\}$. The induction hypothesis in the case $n=m$ lets us select $B_{1}^{*}, \ldots, B_{N_{0}}^{*}$ such that

$$
\begin{gathered}
\bigcup_{\alpha \in J_{0}} B_{\alpha}^{*} \subset \bigcup_{i=1}^{N_{0}}\left(1+C_{m} \delta\right) B_{i}^{*}, \\
N_{0} \leq c \delta^{-(2 m-3) / 4} .
\end{gathered}
$$

Then by Lemmas 2.6, 2.13, and 2.14, we have proved (2.11) and (2.12) and then the claim for $Q_{m+1}^{0}$. Since we can do the same selection in each $Q_{m+1}^{k}$ as we did in $Q_{m+1}^{0}$, we can select $\left\{B_{k_{j}}\right\}$ such that

$$
\begin{gathered}
\bigcup_{\alpha \in J_{k}} B_{\alpha} \subset \bigcup_{j=1}^{N_{k}}\left(1+C_{m+1} \delta\right) B_{k_{j}}, \\
N_{k} \leq c \delta^{-(2 m-3) / 4} .
\end{gathered}
$$

By (2.15) and (2.16) we can select $\left\{B_{k_{j}}\right\}$ such that

$$
\bigcup_{\alpha \in I} B_{\alpha} \subset \bigcup_{k=1}^{l} \bigcup_{j=1}^{N_{k}}\left(1+C_{m+1} \delta\right) B_{k_{j}}
$$

and

$$
\sum_{k=1}^{l} N_{k} \leq l \cdot c \delta^{-(2 m-3) / 4} \leq \delta^{-1 / 2} \cdot c \delta^{-(2 m-3) / 4} \leq c \delta^{-(2(m+1)-3) / 4},
$$

which proves Lemma 2.8 by induction. Q.E.D.

\section{SKetch of the proof of Lemma C}

Once we have Lemma 2.8 , we can do exactly the same proof as in the case $n=2$ (see [L]), and then we have the following lemmas.

Lemma 3.1. Let $\delta$ be given small enough. Let any cube $\mathscr{Q}$ in $R^{n}, n \geq 2$, with sidelength $2^{k} \sqrt{\delta}$ be given, and let $\left\{B_{\alpha}\right\}_{\alpha \in I}$ be any finite collection of balls with $r \leq \rho\left(B_{\alpha}\right) \leq r+2^{k} \delta$ for some $2^{k} \leq r \leq 2^{k+1}$ and centered in $\mathscr{Q}$, where $k$ is an integer. Then one can select a subcollection of balls $B_{1}, \ldots, B_{N}$ such that

$$
\begin{gathered}
\bigcup_{\alpha} B_{\alpha} \subset \bigcup_{i=1}^{N}\left(1+C_{n} \delta\right) B_{i}, \\
N \leq c \delta^{-(2 n-3) / 4},
\end{gathered}
$$

where $C_{n}$ only depends on the dimension $n$ and $c$ is a fixed constant (as in Lemma 2.1).

The proof of Lemma 3.1 is straightforward if we use Lemma 2.8 and the scaling property. Actually, Lemma 3.1 can be reduced to Lemma 2.8 by dilating $R^{n}$ by $2^{-k}$. 
Lemma 3.4. Let $\Delta$ be given small enough. Let any cube $\mathscr{Q}$ in $R^{n}, n \geq 2$, with sidelength $2^{k} \sqrt{\delta}$ be given, and let $\left\{B_{\alpha}\right\}_{\alpha \in I}$ be any finite collection of balls with $2^{k} \leq \rho\left(B_{\alpha}\right) \leq 2^{k+1}$. Then one can select a subcollection $B_{1}, \ldots, B_{N}$ such that

$$
\begin{gathered}
\bigcup_{\alpha} B_{\alpha} \subset \bigcup_{i=1}^{N}\left(1+C_{n} \delta\right) B_{i}, \\
N \leq c \delta^{-(2 n-1) / 4},
\end{gathered}
$$

where $C_{n}$ depends only on the dimension $n$ and $c$ is a fixed constant (as in Lemma 2.1).

The proof of Lemma 3.4 follows the routine of the proof of Lemma 5.2 in [L].

Lemma 3.7. Let $\delta$ be given small enough and $\left\{B_{\alpha}\right\}_{\alpha \in I}$ be a finite collection of balls in $R^{n}, n \geq 2$, with $2^{k} \leq \rho\left(B_{\alpha}\right) \leq 2^{k+1}$, where $k$ are integers. Then one can select balls $B_{1}, \ldots, B_{N}$ such that

$$
\begin{gathered}
\bigcup_{\alpha} B_{\alpha} \subset \bigcup_{i=1}^{N}\left(1+C_{n} \delta\right) B_{i}, \\
\sum_{i=1}^{N} \chi_{B_{i}}(x) \leq c \delta^{-n+1 / 4}
\end{gathered}
$$

for all $x \in R^{n}$, where $C_{n}$ only depends on the dimension $n$ and $c$ is some fuxed constant (as in Lemma 2.1).

The proof follows the proof of Lemma 5.3 in [L]. We subdivide $R^{n}$ into a dyadic grid of $\left\{Q_{j}\right\}_{j=1}^{\infty}$ whose sidelengths are $2^{k} \sqrt{\delta}$. The only difference here is that the cardinality of those special $j$ is $\delta^{-n / 2}$ (which was $\delta^{-1}$ in Lemma 5.3 in [L]).

Finally, we can prove Lemma $C$ by methods similar to the proof of Lemma 1 in [L]. Since the method of proof has nothing to do with the dimension, everything is the same, except we change $R^{2}$ to $R^{n}$.

\section{ACKNOWLEDGMENT}

The author wishes to thank Professor S. Chanillo for his constant encouragement and many useful suggestions and conversations. He is also grateful to Professor B. Muckenhoupt for the helpful discussions with him and to Professor Tom Wolff for his interest in this work.

\section{REFERENCES}

[CM] S. Chanillo and B. Muckenhoupt, Nodal geometry on Riemannian manifolds, J. Differential Geom. 34 (1991), 85-92.

[DF] H. Donnelly and C. Fefferman, Nodal sets of eigenfunctions on Riemannian manifolds, Invent. Math. 93 (1988), 161-183.

[DF2] _ Growth and geometry of eigenfunctions of the Laplacian, Analysis and Partial Differential Equations, Lecture Notes in Pure and Appl. Math., vol. 122, Dekker, New York, 1990. 
[L] G. Lu, Covering lemmas and BMO estimates for eigenfunctions on Riemannian surfaces, Rev. Mat. Iberoamericana 7 (1991), 221-246.

[SW] E. T. Sawyer and R. L. Wheeden, Weighted inequalities for fractional and Poisson integrals in Euclidean and homogeneous spaces, Amer. J. Math. 114 (1992), 813-874.

Department of Mathematics, 253-37, California Institute of Technology, Pasadena, CALIFORNiA 91125

E-mail address: glu@math.rutgers.edu or gzlu@cco.caltech.edu 